\title{
Mallarmé, Valéry et le silence (dés)incarné
}

Les affinités entre les poétiques de Mallarmé et de Valéry ont été envisagées de plusieurs points de vue. Même si ce dernier a deux fois récusé l'influence mallarméenne sur son esthétique, cela ne fait pas de doute qu'il a retenu quelques leçons de son maitre. Voici la problématique de la musicalité littéraire et du silence qui constitue une part importante dans l'écriture de Mallarmé au point de lui inspirer la partition du Coup de dés, et qui réapparaît dans les réflexions de Valéry. Fidèle à la primauté mallarméenne de la forme sur le fond, Valéry part dans sa création des figures rythmiques vides qui relèvent du silence des sons. Mais dans son traitement du silence et des fonctions qu'il lui attribue, le disciple dépasse la pensée du maître. Le silence, qui devait être écrit dans la blancheur de la page, commence à se désincarner dans le pur qui s'approche du sacré.

\section{Mallarmé et la musicalité silencieuse du texte}

\subsection{La musique en train de se taire}

L'originalité de la poétique mallarméenne relève de la nécessité préalable d'établir « la différence entre poésie et poésie » (Gonçalvez, 1999 : 309) en vue de saisir le concept artistique du poète. Dans ses recherches de l'art idéal à déchiffrer par les lecteurs, c'est la musique, à laquelle il voulait "reprendre [son] bien » (« Crise de vers », OC $I I: 212)^{1}$, qui apparaît comme la clé d'interprétation. Mais là se cache un second paradoxe : il faudrait faire la différence entre musique et musique, car l'aboutissement de toutes les opérations mallarméennes, visant la musicalité, conduit au concert silencieux joué par la parole poétique et résonnant dans la conscience du lecteur. Le silence conceptuel se transforme donc en silence textuel ce qu'a bien remarqué Paul Valéry dans son témoignage sur le Coup de dés : "Ma vue avait affaire à des silences

Anna Opiela-Mrozik - docteur ès lettres, maître de conférences à l'Institut d'Études romanes de l'Université de Varsovie. Adresse pour correspondance : Institut d'Études romanes de l'Université de Varsovie, ul. Dobra 55, 00-312 Varsovie, Pologne ; e-mail : am.opiela@uw.edu.pl

1. Tous les renvois aux œuvres analysées de Mallarmé se rapportent à l'édition des Euvres complètes (1998 vol. I. 2003 vol. II. Paris. Gallimard) et seront désormais abrégés OC I et OC II. 
qui auraient pris corps " ("Le Coup de dés. Lettre au diecteur des Marges ", E I : $624)^{2}$. L'espacement du poème illustre cette tentative d'écrire le silence ou de l'incarner au moyen de l' «intellectuelle parole » (OC II : 212).

Si le Coup de dés apparaît comme un aboutissement du projet mallarméen, force est de revoir les étapes d'une transformation progressive de la musicalité littéraire qui ont précédé sa forme la plus sophistiquée. Le rôle de la musique-support dans l'émancipation du mot poétique a été bien analysé par Valéry : dans plusieurs textes qu'il a consacrés à son maitre, on retrouve la nouvelle définition du beau à l'état pur, source d'une sensation esthétique pareille à celle que produit une composition musicale. L'objectif de Mallarmé se ramène donc à « émouvoir par des formes et des objets dont l'art seul fait des forces émouvantes, repousser la simulation, ne se fonder ni sur la crédulité ni sur la niaiserie, ne pas spéculer sur les réactions les plus probables [...] » («Stéphane Mallarmé », E I: 676). Le beau obscur et inattendu chez Mallarmé résulte du travail sur le langage qui a pour but de suggérer au lieu de nommer, et d'exploiter ses ressources formelles et sonores. En reprenant la définition valéryenne du poème, « cette hésitation prolongée entre le son et le sens » («Rhumbs », CE II: 637), la poétique de Mallarmé joue d’abord sur le premier aspect : le vers s'imposant par sa sonorité et non par son sens. Valéry a relevé donc la musicalité de premier niveau ${ }^{3}$ qui, de même que chez Baudelaire, se fonde sur la prosodie. Mais, à en croire Jill Anderson, la sonorité du texte, exploitée dans les poèmes de jeunesse, annonce déjà le passage à la musique intérieure " tissée de sonorités apparentes et virtuelles » ou, autrement dit, le silence conçu selon le Coup de dés : grâce aux « mots en écho suspendus dans la blancheur de la page ", la disposition typographique du Coup de dés se trouve dissimulée au sein des poèmes des années 1860 (2002:4).

Il est pourtant important de noter que le sonore chez Mallarmé prend une nuance péjorative (Tibi, $1998: 214$ ). Dans le fameux sonnet «en -yx », il est question de "l'aboli bibelot d'inanité sonore " (OC $I: 98)$, ce qui marque un abandon progressif de la musicalité II. Les évocations des instruments en train de se taire illustrent l'acheminement mallarméen vers une nouvelle musique que le poète considère dans une "perspective 'scripturale' (Buchs, $2016: 47-48$ ). Dépourvue de sa sonorité, cette musique se dissimule au sein de la parole poétique en visant la «divine transposition » qui va "du fait à l'idéal » ("Théodore de Banville », OC II : 144). Par ailleurs, selon les réflexions notées dans le texte La Musique et les Lettres, la musique ne résonne que pour se dissoudre tout de suite dans le silence. En raison de sa na-

2. Tous les renvois aux œuvres analysées de Valéry se rapportent à l'édition des Euvres (1957 vol. I. 1960 vol. II. Paris. Gallimard) et seront désormais abrégés $E$ I et $E$ II.

3. Nous reprenons ici la typologie des musicalités littéraires proposée par Andrzej Hejmej (2012. Muzyczność dzieła literackiego. Toruń. Wydawnictwo Naukowe UMK. 60-61). Selon ce classement, la musicalité I se rapporte à tous les essais littéraires fondés sur la sonorité du langage, la musicalité II concerne les musiques verbales ou, autrement dit, la thématisation de la musique au sein d'une œuvre littéraire, la musicalité III envisage la transposition des techniques et formes musicales dans la littérature. 
ture fugitive et insaisissable, elle s'identifie à la " dispersion volatile» (OC II : 65) ou «l'état opulent aussitôt évasif » (OC II : 69). Le thème de la musique fonctionne donc toujours selon une dialectique de la présence et de l'absence et c'est dans la «Crise de vers » que Mallarmé semble indiquer le sort de l'art des sons dans son aspiration à l'idéal du langage poétique : « Je dis : une fleur ! et, hors de l'oubli où ma voix relègue aucun contour, en tant que quelque chose d'autre que les calices sus, musicalement se lève, idée même et suave, l'absente de tous bouquets " (OC II : 213). Il s'agirait donc finalement d'" une Musique sans musique » (Buchs, 2016 : 56), n'étant que la représentation muette de la notion pure.

\subsection{Vers le silence-absence}

Dans la poétique mallarméenne, il est possible d'apercevoir une progressive disparition de la musique, prise "dans l'acception ordinaire » de ce terme, et obtenue " avec le secours, des cordes, des cuivres et des bois " (La Musique et les Lettres, OC II : 68). C'est dans la perspective d' " un solitaire tacite concert ( "Le Livre, instrument spirituel », OC II : 226) qui résonne dans l'esprit du lecteur, que Mallarmé œuvrait à élaborer, ce qu'Alain Vaillant a appelé « l'esthétique du rien » $(2005: 376)$ où les objets s'évanouissent pour laisser place à «la toute-puissance du verbe purement poétique » (Albrecht, 2012 : 162). Ce processus, dont l'inspiration vient de la musique même, a été bien interprété par Valéry : "Comme le monde des sons purs, si reconnaissables par l'ouïe, fut extrait du monde des bruits pour s'opposer à lui et constituer le système parfait de la Musique, ainsi voudrait opérer l'esprit poétique sur le langage » (« Je disais quelquefois à Stéphane Mallarmé ", E I: 648). L’acheminement vers la plénitude de la parole se trouve illustré par le "Triptyque de l'Absence " qu'ouvre le poème Apparition. Le concert des êtres spirituels se déroule dans un décor quasi imperceptible et dans une ambiance de tristesse omniprésente qui accompagne l'évanouissement de la sonorité instrumentale :

La lune s'attristait. Des séraphins en pleurs

Rêvant, l'archet aux doigts, dans le calme des fleurs

Vaporeuses, tiraient de mourantes violes

De blancs sanglots glissant sur l'azur des corolles (OC I : 7).

L'évocation de la blancheur indique l'avènement du silence qui semble s'exhaler déjà des "mourantes violes». Il est intéressant de reprendre à ce propos le message du poème Une dentelle s'abolit, même si, du point de vue de la chronologie, il occupe la dernière place dans le Triptyque. C'est le texte de la naissance avortée, expression de la négativité pure que seuls les mots peuvent abolir, tandis que l'instrument reste stérile et muet. De nouveau, sur le fond blanc « Tristement dort une mandore / Au creux néant musicien » $(O C I: 42)$. L'impuissance du poète qu'illustre la métaphore de l'instrument, se trouve cependant vaincue par la parole créatrice : le jeu des sonorités indique une mandore dissimulée au début du vers : «-ment dort». 
Le relais, pris par les mots, face au silence des instruments, voici le message de Sainte, le dernier poème du Triptyque. C'est là que se dessine la rupture définitive avec toute une tradition musicale : viole, flute et mandore, ces attributs de la sainte Cécile, patronne de la musique religieuse, tombent en désuétude avec leur sonorité, peut-être trop bruyante. Ils n'existent qu'au niveau d'une image du passé et leur absence, conjuguée à celle du livre de Magnificat, annonce la transformation de la sainte qui dès lors veillera sur la poésie, cette musique silencieuse selon Mallarmé. Au lieu d'en jouer, sainte Cécile, dont le nom pourrait donner l'anagramme de "silence », se sert du "plumage instrumental " pour écrire. En tant que "musicienne du silence ", elle symbolise le rejet des musicalités I et II au profit de celle d'un ordre nouveau : celle du "poëme tu, aux blancs » («Crise de vers », OC II : 211). Comme le remarque Florent Albrecht, sainte Cécile devient ainsi « l'emblème d'une vision post-hérodiadéenne de la poésie, où la volonté de rupture et d'isolement cède la place à l'éclosion d'une poésie silencieuse » (2016:89-90).

\subsection{L'espacement de la poésie ou l'incarnation du silence}

Force est de mentionner que la scène d'Hérodiade ainsi que L'Après-midi d'un faune marquent déjà un nouveau destin de la poésie et sa marche vers le silence. Les répliques d'Hérodiade et son opposition à la Nourrice, symbole de l'ancienne Poésie, témoignent de ce "mirage interne des mots mêmes " ("À Henri Cazalis ", OC I : 731), rêvé par Mallarmé. " Tout, ici, ne reposait que sur la vertu enchanteresse du langage ", a noté Valéry ("Je disais quelquefois... ", $E I: 649)$. Quant à la flûte du faune, qui émet une "sonore, vaine et monotone ligne" (OC I :24), il s'agit d'un double aspect suggérant l'approche du silence : d'un côté, la musique exécutée par le faune est en train de s'évanouir dans le vide d'un son unique ("vanus » latin veut dire " creux, vide »), mais de l'autre, le jeu se matérialise dans la ligne, cette représentation visuelle et silencieuse de la mélodie. C'est là que se dessine une conception architecturale de la musique dont témoigne la typographie du poème où les blancs s'identifient aux pauses, les majuscules marquent des accents et les parties en italiques annoncent le changement de registre. Par ailleurs, Mallarmé considérait la musicalité III comme la clé de l'interprétation du poème, qui devait contenir " une sorte de jeu courant pianoté autour, comme qui dirait d'un accompagnement musical » ( "Sur l'évolution littéraire », OC II : 701). Valéry parle même d'une " fugue littéraire, où des thèmes s'entrecroisent avec un art prodigieux » ("Stéphane Mallarmé », E I : 670) en relevant ainsi l'existence de la musique au niveau spatial du texte. D'ailleurs, poursuit Valéry, Mallarmé n'était pas satisfait de voir Debussy écrire une partition à son poème, car, "sa musique à lui suffisait "et c'était 'un véritable attentat contre la poésie" " "(ibid.).

Et pourtant, avec son Prélude à l'après-midi d’un faune, Debussy a bien saisi le projet mallarméen de « jouer » en silence avec les moyens poétiques. Les arabesques des sons musicaux reflètent donc ceux des mots imaginés par le poète. Plus encore : l'analyse de la forme et des suggestions interprétatives, dans le cycle de Trois poèmes 
de Stéphane Mallarmé, permet d'apercevoir ces « architectures mobiles musicales " (1946 : 216) auxquelles aspirait l'auteur des textes. Debussy apparait donc comme celui qui a compris le nouveau concept de la musique et du silence qui dépassent le cadre de l'art du temps. En effet, si dans L'Après-midi d'un faune s'opère ce que Mallarmé a appelé " une brisure des grands rythmes littéraires " ("Crise de vers", OC II : 212) conjuguée aux blancs, c'est dans le Coup de dés que le poète s'est complètement libéré du vers et, ce qui en résulte, de la musicalité conçue dans un sens traditionnel. Le Coup de dés instaure une nouvelle idéologie de la musique silencieuse destinée à l'œil, incarnée dans l'organisation typographique de la page. L'enjeu musical passe donc du temporel au spatial et se déroule "selon une vision simultanée de la Page " (OC I : 391). Se réalise ainsi le vœu mallarméen du Mystère dans les Lettres : le Coup de dés ne fait qu' "authentiquer le silence " (OC II : 234). C'est l'espace qui crée le sens et concourt à l'expression de la pensée parfaite, tout en agissant sur le lecteur par son silence même. Valéry se souvient de

voir la figure d'une pensée, pour la première fois placée dans notre espace... Ici véritablement l'étendue parlait, songeait, enfantait des formes temporelles. [...] [S]a vue avait affaire à des silences qui auraient pris corps. [...] Cétaient, murmures, insinuations, tonnerres pour les yeux ( Le Coup de dés... », $E I: 624$ ).

Il est important d'ajouter que pour Mallarmé le phénomène de l'incarnation du silence coïncide, voire s'identifie, avec l'exécution de la musique intellectuelle des mots, cet " ensemble des rapports existant dans tout » "Crise de vers », OC II : 212). Le triomphe du silence n'est autre que celui de la parole :

Je fais de la Musique, et appelle ainsi non celle qu'on peut tirer du rapprochement euphonique des mots [...], mais l'au-delà magiquement produit par certaines dispositions de la parole [...]. Vraiment entre les lignes et au-dessus du regard cela se passe, en toute pureté, sans l'entremise de cordes à boyaux et de pistons comme à l'orchestre, qui est déjà industriel ; mais c'est la même chose que l'orchestre, sauf que littérairement ou silencieusement («À Edmond Gosse », OC I : 807).

\section{Valéry et le silence dans tous ses états}

Chez Paul Valéry réapparaît une réflexion sur le silence, inhérente à celle sur la musicalité du texte et dans le texte. Une question se pose : quelle fonction le poète, ce disciple de Mallarmé, attribue-t-il à la musique et au silence ? Tout d'abord, il faut souligner que dans son idée de la poésie Valéry se référait constamment à la musique - d'où le nombre de termes musicaux et la recherche des analogies entre les deux modes dexpression. Cependant, l'acception valéryenne de la musique n'est pas celle de l'art des sons, tout au 
contraire : elle s'élève au niveau des pures abstractions, d'une « sonorité » intérieure qui fait ainsi relever le sens du silence : « La musique qui est en moi, / La musique qui est dans le silence, en puissance / qu'elle vienne et m'étonne» (C II: 1267) .

Dans l'esthétique valéryenne le silence constitue un point de départ, un fond non moins important que les sons où ceux-ci s'établissent. Mais, dans un sens philosophique, le terme de Silence renvoie également à ce qui suit le résonnement des sons, illustrant ainsi « la continuité de la fonction auditive » $(C I: 1155)$.

La valorisation du silence, cet élément constitutif du rythme musical et poétique, coïncide avec une appréhension progressive de la musique qui, paradoxalement, séloigne du son, au profit d'un symbole et de l'expression d'une pensée abstraite. Tout en se muant vers le pur, la musique s'avère d'autant plus féconde pour la poésie dont l'objectif est de réussir cette alliance intime du son et du sens. D’ailleurs, à en croire l'expérience vécue par Valéry, un jour, lors d'une promenade, il a été saisi d'une intuition musicale qui depuis s'est installée dans sa conscience du poète. Sans être musicien, c'est par le biais de la musique que le poète a vu se poser un univers à lui « avec tous ses rapports et ses proportions » ( $E I: 1327)$.

\subsection{Le silence incarné dans l'architecture}

La rencontre de la Musique et de la Poésie s'effectue, chez Valéry, de même que chez son maitre, à travers l'idée de la forme. Si l'univers poétique s'appuie sur des relations entre les mots, dans la musique, ce sont des « unités sonores » qui « sont aptes à former des combinaisons claires, des implications successives ou simultanées, des enchaînements et des croisements que l'on peut dire intelligibles : c'est pourquoi il existe en musique des possibilités abstraites " ("Variété », $E I: 1326)$. La musique conçue en terme de structure, au détriment de sa sonorité, fait penser à la musicalité III, ce que confirme une dimension architectonique dont le poète dote l'art des sons. C'est le destin commun et les relations intrinsèques qui rapprochent ces deux arts : « Il est clair que musique et architecture sont des arts qui se passent également de l'imitation des choses ; ce sont des arts dans lesquels la matière et la forme ont des relations beaucoup plus intimes entre elles que dans les autres » ("Histoire d'Amphion », $C E$ $I I$ : 1279), etc. En raison de cette affinité " entre deux incarnations de l'art " (Notes, $E$ II : 1405), dans l'esthétique valéryenne, une œuvre architectonique peut être décrite avec les termes propres à la musique. Le silence, cet élément constitutif de l'art musical considéré comme une structure spatiale, s'incarne donc dans l'architecture. Selon une vision d'inspiration baudelairienne, l'intérieur du temple s'identifie à « la forêt du silence... » et « c'est la forêt où l'on oublie, où l'on écoute !» (ibid.). À l'arabesque mallarméenne succède "un espace intelligible et changeant » ("Eupalinos », E II : 102) comme celui du Cantique des colonnes où les images musicales se confondent avec les évocations architectoniques :

4. Tous les renvois aux notes des Cahiers de Valéry se rapportent à l'édition de Judith Robinson (1973 vol. I. 1974 vol. II. Paris. Gallimard) et seront désormais abrégés C I et C II. 
Douces colonnes, ô

L'orchestre des fuseaux!

Chacun immole son

Silence à l'unisson ( $E I: 116)$.

Dans l'esthétique de Valéry, la musique se fonde sur des combinaisons silencieuses qui abolissent la sonorité comme son élément constitutif : « le son ne contient pas la musique ; elle ne sort pas nécessairement de l'existence des sons. Et réciproquement l'essentiel de la musique ne demande pas le son " (C II : 938). Il s'agit donc de la musique qui, à en croire l'expérience de Valéry, fait oublier à l'auditeur les sensations perçues par l'oreille pour l'inviter ensuite à une prise de conscience du Moi, à une relation de soi à soi où n'intervient aucune référence à la réalité extérieure. C'est la naissance du chant silencieux de la vie intérieure qui n'est entendu que par l' "ouïe intime » dont procède tout acte créateur (Stimpson, 1993 : 52).

\subsection{Les modulations en vue de l'art pur}

À force de cotôyer constamment l'univers musical, l'univers poétique de Valéry s'appuie sur un principe à deux versants apparemment contradictoires : les structures et procédés formels complétés d'une dimension abstraite, voire psychique. Car la musicalité valéryenne, encore plus que chez Mallarmé où tout se joue dans l'esprit, engage non seulement l'intellect, mais aussi toute la vie affective et spirituelle de l'individu. Le silence apparaît donc comme un facteur nécessaire dans la quête du Moi. Quelle qu'elle soit, (architectonique ou non), la Musique de Valéry, tout en se passant de musique, tend plutôt à « désincarner » le silence à travers un élargissement de l'acception des termes comme " voix " ou " modulation » au domaine psychologique. L'idée de la voix, en effet, se place au centre de la pensée valéryenne : comme il note dans les Cahiers, " on fait les vers de sa voix » (C II : 1094), où la " voix » devient le concept le plus original dans la poétique de Valéry qui renvoie à une dimension psychologique de la parole :

La plus belle poésie à la voix d'une femme idéale, Mlle Âme. Pour moi, la voix intérieure me sert de repère. [...] car la voix intérieure ne supporte que les paroles dont le sens est secrètement d'accord avec l'être vrai ; dont la musique est le graphique même des mouvements et arrêts de cet être (C II, 1076).

La voix silencieuse s'identifierait donc à une modalité de l'écoute interne du poète, contrairement à " la disparition élocutoire » de celui-ci, prônée par Mallarmé. C'est aussi dans le silence que se jouent les "modulations psycho-physiques", une sorte de glissement d'un état à l'autre (Suhami, $1966: 48$ ) qui, à en croire Valéry, a guidé la création de La Jeune Parque (E I : 1473). Ce poème retrace les états successifs de la conscience en mouvement perpétuel, ainsi que la soif de la pureté inhérente au silence : «Et que de mes destins lentements divisé, / Le plus pur en silence éclaire 
un cœur brisé » (CE I: 96); «Femme flexible et ferme aux silences suivis / D'actes purs !... » (E I : 99). Le motif du silence ou du non-dit acquiert, dans l'esthétique valéryenne, la valeur d'un message de la sensibilité. Aussi la jeune Parque se demandet-elle dans l'intimité de son être : "Et quel silence parle à mon seul possesseur ? » (E I : 97). Mais dans les dernières séquences du poème, Valéry confirme le caracère « désincarné » du silence évoqué dans le contexte spirituel :

Où l'âme, ivre de soi, de silence et de gloire,

Prête à sévanouir de sa propre mémoire,

Écoute, avec espoir, frapper au mur pieux

Ce cour [...] (E I : 107).

Si dans sa quête du moi la jeune Parque avoue partir du rien, étant « de [s]on néant secrètement armée » ( $E I: 100)$, elle indique ainsi la genèse silencieuse de son aventure intérieure. De même, la forme musicale, qui sort du néant, se trouve à l'origine du Cimetière marin. Selon Mémoires du Poète, l'intention de Valéry était de créer un poème à partir d' " une figure rythmique vide, ou remplie de syllabes vaines, qui [1]e vint obséder quelque temps » (E I: 1503). L'apparition de la structure strophique est anticipée par la musique réduite à un rien, cette expression de l'absence et l'image du silence. Il faut que cette musique initiale, toute muette qu'elle soit, "creuse le lit spirituel " (C I : 199) des vers futurs. Les évocations du silence, dans Le Cimetière marin, confirment son aspect "pur » et le passage au paysage intérieur : « Ô mon silence !... Édifice dans l'âme » (EI:148). D’ailleurs, l'image de la mer et de son bruit continu se ramène finalement à une espèce de silence (" dans un tumulte au silence pareil », $E I: 151)$, celle à laquelle « répond le beau. Ou le vers pur, ou l'idée lumineuse » (EI:1449). Et c'est là que réside la perfection de ce poème harmonique et mélodieux à merveille.

\section{3. Entre présence et absence : deux filles du silence}

La métaphore du silence illustre un enjeu de la poétique valéryenne visant le pur, ainsi que la tension entre l'art et le désir dont témoigne le poème Les pas construit autour de l'idée du silence. En tant qu' " un élément positif-productif-transitif » (C II : 974), le silence fait naître le poème, mais aussi apparaît comme son point d'aboutissement. La première strophe instaure une présence-absence quasi sensible, sortie du néant :

Tes pas, enfants de mon silence,

Saintement, lentement placés,

Vers le lit de ma vigilance

Procèdent muets et glacés $(E I: 120)$.

L'ambiance et le décor de cette apparition spontanée et silencieuse font penser plutôt à la mort qu'à l'amour. La deuxième strophe confirme le lien entre le silence 
et le pur, voire le sacré, car l'inconnue rappelle un être supraterrestre, «ombre divine ", et porteuse de dons spirituels. Le poème pose ensuite la question du désir : c'est le désir des pas qui se ramène, à la fin, à celui du désir de l'autre : "Car j'ai vécu de vous attendre / Et mon cœur n'était que vos pas » (E I: 121). Comme dans le poème mallarméen Une dentelle s'abolit..., la situation intiale se renverse, car ce que le « je » a engendré (" les pas») se trouve à l'origine du " je». Le silence, qui semble tout d'abord "s'incarner » dans les pas, ce signe de la présence, s'avère se muer en désir de la présence-absence dont l'image serait une venue retardée : " $\mathrm{Ne}$ hâte pas cet acte tendre, / Douceur d'être et de n'être pas " (ibid.). Comme le remarque Maria Watroba, «si la présence en elle-même est plénitude, ce qu'elle annonce, c'est nécessairement l'absence (de l'autre); et du coup, elle devient absence à soi-même » (2007 : 133). C'est donc le retour au silence, cette fois-ci le silence ultime, symbolisant le néant spirituel ou l'au-delà dont est sortie l'inconnue (une allégorie de la mort ?) et où elle veut entraîner le « je » qui l'attend et ne l'attend pas dans son lit « de vigilance».

\subsection{La musique tacite et le silence désincarné}

Certes, dans l'œuvre de Valéry, il est possible de trouver le traitement mallarméen de la page selon lequel les blancs séparant les parties du texte ont pour but d' "incarner " le silence, tandis que les accents dans la musique du poème sont marqués par les mots en majuscules comme MOI de La Jeune Parque. Cependant, dans la quête de l'art pur, le silence devient un outil dépassant les ressources du langage et, dans son acception symbolique et par cela "désincarnée ", il rejoint le sacré. Telle est l'idée qui se dégage de l'étude des Cantiques spirituels où Valéry partage ses réflexions que lui a inspirées la lecture des ouvrages spirituels de saint Jean de la Croix. Même si Valéry affirme ne pas se connaître à la mystique, sa compréhension des textes de saint Jean est des bien profondes. L'analyse valéryenne établit d'emblée une dimension musicale des Cantiques spirituels : ce sont les " harmoniques de [1] a pensée mystique " en forme d' " un chant très tendre " ( $E I$ : 448-449). C'est une mélodie charmante du langage que le traducteur a réussi à dégager du silence à la manière des maîtres : «Est-il, dans La Fontaine même, ou dans Verlaine, chant plus fluide, fluide, mais pas lâche, évadé plus heureusement du silence? " (E I : 451-452). Dans cette poésie qui donne à Valéry un sentiment de perfection, c'est l'âme qui se parle à elle-même "entre deux silences absolus ", dans la «solitude sonore» du langage (Allain-Castrillo, 1993 : 267). Ces silences semblent renvoyer, d'un côté, au silence nécessaire dans l'appréhension mystique de Dieu, et de l'autre, à la puissance et à l'économie du langage grâce auxquelles s'est réalisée l'alliance intime du son et du sens.

Accompagné d'une "musique tacite " des mots, Valéry, lecteur des Cantiques spirituels, avance, dans la " Nuit obscure ", le thème préféré de saint Jean de la Croix, thème qui nécessite la présence du silence, ce symbole de la foi. Valéry, quant à lui, parle de l'«obscurité-silence chargée » (C II : 978), un état d'attente, précédant l'ou- 
verture au mystère de la foi ou de l'art, l'apparition d'une ouïe spirituelle. S'il en résulte une musique intime, elle est vouée au Silence final qui prolonge la volonté d'entendre. Par ailleurs, la beauté de l'art réside dans sa capacité de réduire le lecteur au silence.

La musique sans musique devient un modèle artistique pour Mallarmé et Valéry. Si le premier a essayé de fondre l'art musical dans celui des mots en incarnant le silence dans l'espace du poème dont témoigne le Coup de dés, l'autre a approfondi la réflexion sur le silence de façon à élargir le projet mallarméen. Le silence du concert joué par l'intellectuelle parole et écouté dans l'esprit s'est transformé en concept-clé de la poétique valéryenne. En tant qu'une notion philosophique, il illustre la vie de l'esprit dont dérive une affinité mystérieuse entre la poésie et la subjectivité. Complété d'un sens mystique, le Silence « désincarné » accompagne le devenir de la Pensée et contribue à l'apparition du Beau pur et abstrait.

\section{BIBLIOGRAPHIE}

Albrecht F. 2016. L'objet musical chez Mallarmé, instrument des fuites. In Bonnet A. \& Frangne P.-H. Mallarmé et la musique, la musique et Mallarmé. PUR. 79-96.

Albrecht F. 2012. Ut musica poesis. Modèle musical et enjeux poétiques de Baudelaire à Mallarmé. Paris. Honoré Champion.

Allain-Castrillo M. 1993. Musique tacite, solitude sonore. Valéry et saint Jean de la Croix. In Stimpson B. \& Gifford P. Paul Valéry: musique, mystique, mathématique. PUL. 267-275.

Anderson J. 2002. La Poésie éprise d'elle-même. Poétique de Stéphane Mallarmé. New York. Peter Lang.

Buchs A. 2016. Une pensée du langage. In Bonnet A. \& Frangne P.-H. Mallarmé et la musique, la musique et Mallarmé. PUR. 47-58.

Gonçalvez A. J. 1999. Stéphane Mallarmé, Paul Valéry : une pensée abstraite. In Bourjea S. Mallarmé/Valéry: poétiques. Bulletin des Études Valéryennes, nº spécial 81/82. 307-321.

Mallarmé S. 1998 (vol. I). 2003 (vol. II). Euvres complètes. B. Marchal (éd.). Paris. Gallimard. Mallarmé S. 1946. Propos sur la poésie. H. Mondor (éd.). Monaco. Éditions du Rocher.

Stimpson B. 1993. «Toute la modulation de lêtre ». La musique qui est en moi. In Stimpson B. \& Gifford P. Paul Valéry: musique, mystique, mathématique. PUL. 37-57.

Suhami E. 1966. Paul Valéry et la musique. Université de Dakar.

Tibi L. 1998. Mallarmé ou le tumulte des sonorités transfusibles en du songe. In F.-Ch. Gaudard. Poésies. Stéphane Mallarmé. Paris. Ellipses. 205-222.

Vaillant A. 2005. La crise de la littérature. Romantisme et modernité. Grenoble. ELLUG.

Valéry P. 1973 (vol. I). 1974 (vol. II). Cahiers. J. Robinson (éd.). Paris. Gallimard. Valéry P. 1957 (vol. I). 1960 (vol. II). CEuvres. J. Hytier (éd.). Paris. Gallimard.

Watroba M. 2007. Écritures de l'absence. PUR. 


\section{Mallarmé, Valéry and (non-)incarnated silence}

ABSTRACT: This article analyses the essence and roles of silence in the ouvres of Mallarmé and Valéry. The Mallarmé's musicality gradually gives way to the silent music of words; poetry is becoming spatial and takes the form of the music score. In the poem Coup de dés it gets up to the incarnation of silence which shows the ideal of the Mallarmé's musicality; the ideal in which an eye serves to listen. The theme of music and silence reappears in the Valery's œuvre. In spite of the connection between music and architecture, which can incarnate silence, poetry uses the subjectivism of music and the ambiguity of silence to achieve the ideal of pure art which is hidden in the artist's interior. Silence as nothingness is the starting point for poetry whereas non-incarnated silence can express the mystical sense of poetry.

Keywords: music, silence, absence, poetry. 